\title{
Guided Unfoldings for Finding Loops in Standard Term Rewriting
}

\author{
Étienne Payet \\ LIM, Université de La Réunion, France \\ etienne.payet@univ-reunion.fr
}

\begin{abstract}
In this paper, we reconsider the unfolding-based technique that we have introduced previously for detecting loops in standard term rewriting. We modify it by guiding the unfolding process, using disagreement pairs in rewrite rules. This results in a partial computation of the unfoldings, whereas the original technique consists of a thorough computation followed by a mechanism for eliminating some rules. We have implemented this new approach in our tool NTI and conducted successful experiments on a large set of term rewrite systems.
\end{abstract}

Keywords: term rewrite systems, dependency pairs, non-termination, loop, unfolding

\section{Introduction}

In [13, we have introduced a technique for finding loops (a periodic, special form of non-termination) in standard term rewriting. It consists of unfolding the term rewrite system (TRS) $\mathcal{R}$ under analysis and of performing a semi-unification [10] test on the unfolded rules for detecting loops. The unfolding operator $U_{\mathcal{R}}$ which is applied processes both forwards and backwards and considers every subterm of the rules to unfold, including variable subterms.

Example 1. Let $\mathcal{R}$ be the TRS consisting of the following rules ( $x$ is a variable):

$$
R_{1}=\underbrace{\mathrm{f}(\mathrm{s}(0), \mathrm{s}(1), x)}_{l} \rightarrow \underbrace{\mathrm{f}(x, x, x)}_{r} \quad R_{2}=\mathrm{h} \rightarrow 0 \quad R_{3}=\mathrm{h} \rightarrow 1 .
$$

Note that $\mathcal{R}$ is a variation of a well-known example by Toyama 18. Unfolding the subterm 0 of $l$ backwards with the rule $R_{2}$, we get the unfolded rule $U_{1}=$ $\mathrm{f}(\mathrm{s}(\mathrm{h}), \mathrm{s}(1), x) \rightarrow \mathrm{f}(x, x, x)$. Unfolding the subterm $x$ (a variable) of $l$ backwards with $R_{2}$, we get $U_{2}=\mathrm{f}(\mathrm{s}(0), \mathrm{s}(1), \mathrm{h}) \rightarrow \mathrm{f}(0,0,0)$. Unfolding the first (from the left) occurrence of $x$ in $r$ forwards with $R_{2}$, we get $U_{3}=\mathrm{f}(\mathrm{s}(0), \mathrm{s}(1), \mathrm{h}) \rightarrow \mathrm{f}(0, \mathrm{~h}, \mathrm{~h})$. We have $\left\{U_{1}, U_{2}, U_{3}\right\} \subseteq U_{\mathcal{R}}(\mathcal{R})$. Now, if we unfold the subterm 1 of $U_{1}$ backwards with $R_{3}$, we get $\mathrm{f}(\mathrm{s}(\mathrm{h}), \mathrm{s}(\mathrm{h}), x) \rightarrow \mathrm{f}(x, x, x)$, which is an element of $U_{\mathcal{R}}\left(U_{\mathcal{R}}(\mathcal{R})\right)$. The left-hand side $l_{1}$ of this rule semi-unifies with its right-hand side $r_{1}$ i.e., $l_{1} \theta_{1} \theta_{2}=r_{1} \theta_{1}$ for the substitutions $\theta_{1}=\{x / \mathrm{s}(\mathrm{h})\}$ and $\theta_{2}=\{\}$. Therefore, 
$l \theta_{1}=\mathrm{f}(\mathrm{s}(\mathrm{h}), \mathrm{s}(\mathrm{h}), \mathrm{s}(\mathrm{h}))$ loops with respect to $\mathcal{R}$ because it can be rewritten to itself using the rules of $\mathcal{R}$ (the redex is underlined at each step):

$$
\mathrm{f}(\mathrm{s}(\underline{\mathrm{h}}), \mathrm{s}(\mathrm{h}), \mathrm{s}(\mathrm{h})) \underset{R_{2}}{\rightarrow} \mathrm{f}(\mathrm{s}(0), \mathrm{s}(\underline{\mathrm{h}}), \mathrm{s}(\mathrm{h})) \underset{R_{3}}{\rightarrow} \underline{\mathrm{f}(\mathrm{s}(0), \mathrm{s}(1), \mathrm{s}(\mathrm{h}))} \underset{R_{1}}{\rightarrow} \mathrm{f}(\mathrm{s}(\mathrm{h}), \mathrm{s}(\mathrm{h}), \mathrm{s}(\mathrm{h})) .
$$

Iterative applications of the operator $U_{\mathcal{R}}$ result in a combinatorial explosion which significantly limits the approach. In order to reduce it, a mechanism is introduced in 13 for eliminating unfolded rules which are estimated as useless for detecting loops. Moreover, in practice, three analyses are run in parallel (in different threads): one with forward unfoldings only, one with backward unfoldings only and one with forward and backward unfoldings together.

So, the technique of [13] roughly consists in computing all the rules of $U_{\mathcal{R}}(\mathcal{R})$, $U_{\mathcal{R}}\left(U_{\mathcal{R}}(\mathcal{R})\right), \ldots$ and removing some useless ones, until the semi-unification test succeeds on an unfolded rule or a time limit is reached. Therefore, this approach corresponds to a breadth-first search for a loop, as the successive iterations of $U_{\mathcal{R}}$ are computed thoroughly, one after the other. However, it is not always necessary to compute all the elements of each iteration of $U_{\mathcal{R}}$. For instance, in Ex. 11 above, $U_{2}$ and $U_{3}$ do not lead to an unfolded rule satisfying the semiunification criterion. This is detected by the eliminating mechanism of 13 , but only after these two rules are generated. In order to avoid the generation of these useless rules, one can notice that $l$ and $r$ differ at the first argument of $\mathrm{f}$ : in $l$, the first argument is $\mathrm{s}(0)$ while in $r$ it is $x$. We say that $\langle\mathrm{s}(0), x\rangle$ is a disagreement pair of $l$ and $r$. Hence, one can first concentrate on resolving this disagreement, unfolding this pair only, and then, once this is resolved, apply the same process to another disagreement pair.

Example 2 (Ex. 1 continued). There are two ways to resolve the disagreement pair $\langle\mathrm{s}(0), x\rangle$ of $l$ and $r$ (i.e., make it disappear).

The first way consists in unifying $\mathrm{s}(0)$ and $x$, i.e., in computing $R_{1} \theta$ where $\theta$ is the substitution $\{x / \mathrm{s}(0)\}$, which gives $V_{0}=\mathrm{f}(\mathrm{s}(0), \mathrm{s}(1), \mathrm{s}(0)) \rightarrow \mathrm{f}(\mathrm{s}(0), \mathrm{s}(0), \mathrm{s}(0))$. The left-hand side of $V_{0}$ does not semi-unify with its right-hand side.

The other way is to unfold $\mathrm{s}(0)$ or $x$. We decide not to unfold variable subterms, hence we select $\mathrm{s}(0)$. As it occurs in the left-hand side of $R_{1}$, we unfold it backwards. The only possibility is to use $R_{2}$, which results in

$$
V_{1}=\mathrm{f}(\mathrm{s}(\mathrm{h}), \mathrm{s}(1), x) \rightarrow \mathrm{f}(x, x, x) .
$$

Note that this approach only generates two rules $\left(V_{0}\right.$ and $\left.V_{1}\right)$ at the first iteration of the unfolding operator. In comparison, the approach of 13 produces 14 rules (before elimination), as all the subterms of $R_{1}$ are considered for unfolding.

Hence, the disagreement pair $\langle\mathrm{s}(0), x\rangle$ has been replaced with the disagreement pair $\langle\mathrm{s}(\mathrm{h}), x\rangle$ in $V_{1}$. Unifying $\mathrm{s}(\mathrm{h})$ and $x$ i.e., computing $V_{1} \theta^{\prime}$ where $\theta^{\prime}$ is the substitution $\{x / \mathrm{s}(\mathrm{h})\}$, we get $V_{1}^{\prime}=\mathrm{f}(\mathrm{s}(\mathrm{h}), \mathrm{s}(1), \mathrm{s}(\mathrm{h})) \rightarrow \mathrm{f}(\mathrm{s}(\mathrm{h}), \mathrm{s}(\mathrm{h}), \mathrm{s}(\mathrm{h}))$. So, the disagreement $\langle\mathrm{s}(0), x\rangle$ is solved: it has been replaced with $\langle\mathrm{s}(\mathrm{h}), \mathrm{s}(\mathrm{h})\rangle$. Now, $\langle 1, \mathrm{~h}\rangle$ is a disagreement pair in $V_{1}^{\prime}$ (here we mean the second occurrence of $\mathrm{h}$ in the right-hand side of $V_{1}^{\prime}$ ). Unfolding 1 backwards with $R_{3}$, we get $W=$ $\mathrm{f}(\mathrm{s}(\mathrm{h}), \mathrm{s}(\mathrm{h}), \mathrm{s}(\mathrm{h})) \rightarrow \mathrm{f}(\mathrm{s}(\mathrm{h}), \mathrm{s}(\mathrm{h}), \mathrm{s}(\mathrm{h}))$ and unfolding $\mathrm{h}$ forwards with $R_{3}$, we get 
$W^{\prime}=f(s(h), s(1), s(h)) \rightarrow f(s(h), s(1), s(h))$. The semi-unification test succeeds on both rules: we get the looping terms $f(s(h), s(h), s(h))$ and $f(s(h), s(1), s(h))$ from $W$ and $W^{\prime}$, respectively.

In the approach sketched in Ex. 2, the iterations of $U_{\mathcal{R}}$ are not thoroughly computed because only some selected disagreement pairs are considered for unfolding, unlike in our previous approach [13] which tries to unfold all the subterms in rules. Hence, now the unfoldings are guided by disagreement pairs. In this paper, we formally describe the intuitions presented above (Sect. 35). We also report experiments on a large set of rewrite systems from the TPBD [17 (Sect 6). The results we get in practice with the new approach are better than those obtained with the approach of [13] and we do not need to perform several analyses in parallel, nor to unfold variable subterms, unlike [13.

\section{Preliminaries}

If $Y$ is an operator from a set $E$ to itself, then for any $e \in E$ we let

$$
(Y \uparrow 0)(e)=e \quad \text { and } \quad \forall n \in \mathbb{N}:(Y \uparrow n+1)(e)=Y((Y \uparrow n)(e)) .
$$

We refer to 4 for the basics of rewriting. From now on, we fix a finite signature $\mathcal{F}$ together with an infinite countable set $\mathcal{V}$ of variables with $\mathcal{F} \cap \mathcal{V}=\emptyset$. Elements of $\mathcal{F}$ (symbols) are denoted by $\mathrm{f}, \mathrm{g}, \mathrm{h}, 0,1, \ldots$ and elements of $\mathcal{V}$ by $x, y, z, \ldots$ The set of terms over $\mathcal{F} \cup \mathcal{V}$ is denoted by $\mathcal{T}(\mathcal{F}, \mathcal{V})$. For any $t \in$ $\mathcal{T}(\mathcal{F}, \mathcal{V})$, we let $\operatorname{root}(t)$ denote the root symbol of $t$ : $\operatorname{root}(t)=\mathrm{f}$ if $t=\mathrm{f}\left(t_{1}, \ldots, t_{m}\right)$ and $\operatorname{root}(t)=\perp$ if $t \in \mathcal{V}$, where $\perp$ is a new symbol not occurring in $\mathcal{F}$ and $\mathcal{V}$. We let $\operatorname{Var}(t)$ denote the set of variables occurring in $t$ and $\operatorname{Pos}(t)$ the set of positions of $t$. For any $p \in \operatorname{Pos}(t)$, we write $\left.t\right|_{p}$ to denote the subterm of $t$ at position $p$ and $t[p \leftarrow s]$ to denote the term obtained from $t$ by replacing $\left.t\right|_{p}$ with a term $s$. For any $p, q \in \operatorname{Pos}(t)$, we write $p \leq q$ iff $p$ is a prefix of $q$ and we write $p<q$ iff $p \leq q$ and $p \neq q$. We also define the set of non-variable positions of $t$ which either are a prefix of $p$ or include $p$ as a prefix:

$$
\operatorname{NPos}(t, p)=\left\{q \in \operatorname{Pos}(t)|q \leq p \vee p \leq q, t|_{q} \notin \mathcal{V}\right\} .
$$

A disagreement position of terms $s$ and $t$ is a position $p \in \operatorname{Pos}(s) \cap \operatorname{Pos}(t)$ such that $\operatorname{root}\left(\left.s\right|_{p}\right) \neq \operatorname{root}\left(\left.t\right|_{p}\right)$ and, for every $q<p, \operatorname{root}\left(\left.s\right|_{q}\right)=\operatorname{root}\left(\left.t\right|_{q}\right)$. The set of disagreement positions of $s$ and $t$ is denoted as $\operatorname{DPos}(s, t)$. A disagreement pair of $s$ and $t$ is an ordered pair $\left\langle\left. s\right|_{p},\left.t\right|_{p}\right\rangle$ where $p \in D \operatorname{Pos}(s, t)$.

Example 3. Let $s=\mathrm{f}(\mathrm{s}(0), \mathrm{s}(1), y), t=\mathrm{f}(x, x, x), p_{1}=1, p_{2}=2$ and $p_{3}=3$. Then, $\left\{p_{1}, p_{2}\right\} \subseteq \operatorname{DPos}(s, t)$ and $\left\langle\left. s\right|_{p_{1}},\left.t\right|_{p_{1}}\right\rangle=\langle\mathrm{s}(0), x\rangle$ and $\left\langle\left. s\right|_{p_{2}},\left.t\right|_{p_{2}}\right\rangle=\langle\mathrm{s}(1), x\rangle$ are disagreement pairs of $s$ and $t$. However, $p_{3} \notin \operatorname{DPos}(s, t)$ because $\left\langle\left. s\right|_{p_{3}},\left.t\right|_{p_{3}}\right\rangle=$ $\langle y, x\rangle$ and $\operatorname{root}(y)=\operatorname{root}(x)=\perp$.

We write substitutions as sets of the form $\left\{x_{1} / t_{1}, \ldots, x_{n} / t_{n}\right\}$ denoting that for each $1 \leq i \leq n$, variable $x_{i}$ is mapped to term $t_{i}$ (note that $x_{i}$ may occur 
in $t_{i}$ ). The empty substitution (identity) is denoted by $i d$. The application of a substitution $\theta$ to a syntactic object $o$ is denoted by $o \theta$. We let $m g u(s, t)$ denote the (up to variable renaming) most general unifier of terms $s$ and $t$. We say that $s$ semi-unifies with $t$ when $s \theta_{1} \theta_{2}=t \theta_{1}$ for some substitutions $\theta_{1}$ and $\theta_{2}$.

A rewrite rule (or rule) over $\mathcal{F} \cup \mathcal{V}$ has the form $l \rightarrow r$ with $l, r \in \mathcal{T}(\mathcal{F}, \mathcal{V})$, $l \notin \mathcal{V}$ and $\operatorname{Var}(r) \subseteq \operatorname{Var}(l)$. A term rewriting system (TRS) over $\mathcal{F} \cup \mathcal{V}$ is a finite set of rewrite rules over $\mathcal{F} \cup \mathcal{V}$. Given a TRS $\mathcal{R}$ and some terms $s$ and $t$, we write $s \rightarrow \overrightarrow{\mathcal{R}} t$ if there is a rewrite rule $l \rightarrow r$ in $\mathcal{R}$, a substitution $\theta$ and $p \in \operatorname{Pos}(s)$ such that $\left.s\right|_{p}=l \theta$ and $t=s[p \leftarrow r \theta]$. We let $\underset{\mathcal{R}}{\stackrel{+}{\rightarrow}}($ resp. $\underset{\mathcal{R}}{\stackrel{*}{\rightarrow}}$ ) denote the transitive (resp. reflexive and transitive) closure of $\overrightarrow{\mathcal{R}}$. We say that a term $t$ is non-terminating with respect to (w.r.t.) $\mathcal{R}$ when there exist infinitely many terms $t_{1}, t_{2}, \ldots$ such that $t \underset{\mathcal{R}}{\rightarrow} t_{1} \underset{\mathcal{R}}{\rightarrow} t_{2} \underset{\mathcal{R}}{\rightarrow}$. We say that $\mathcal{R}$ is non-terminating if there exists a non-terminating term w.r.t. it. A term $t$ loops w.r.t. $\mathcal{R}$ when $t \underset{\mathcal{R}}{+} C[t \theta]$ for some context $C$ and substitution $\theta$. Then $t \underset{\mathcal{R}}{\stackrel{+}{\rightarrow}} C[t \theta]$ is called a loop for $\mathcal{R}$. We say that $\mathcal{R}$ is looping when it admits a loop. If a term loops w.r.t. $\mathcal{R}$ then it is non-terminating w.r.t. $\mathcal{R}$.

The unfolding operators that we define in Sect. 3 of this paper use narrowing. We say that a term $s$ narrows forwards (resp. backwards) to a term $t$ w.r.t. a TRS $\mathcal{R}$ when there exists a non-variable position $p$ of $s$ and a rule $l \rightarrow r$ of $\mathcal{R}$ renamed with new variables not previously met such that $t=s[p \leftarrow r] \theta$ (resp. $t=s[p \leftarrow l] \theta)$ where $\theta=m g u\left(\left.s\right|_{p}, l\right)$ (resp. $\theta=m g u\left(\left.s\right|_{p}, r\right)$ ).

We refer to [3] for details on dependency pairs. The defined symbols of a TRS $\mathcal{R}$ over $\mathcal{F} \cup \mathcal{V}$ are $\mathcal{D}_{\mathcal{R}}=\{\operatorname{root}(l) \mid l \rightarrow r \in \mathcal{R}\}$. For every $\mathrm{f} \in \mathcal{F}$ we let $\mathrm{f} \#$ be a fresh tuple symbol with the same arity as $\mathrm{f}$. The set of tuple symbols is denoted as $\mathcal{F}^{\#}$. The notations and definitions above with terms over $\mathcal{F} \cup \mathcal{V}$ are naturally extended to terms over $(\mathcal{F} \cup \mathcal{F} \#) \cup \mathcal{V}$. Elements of $\mathcal{F} \cup \mathcal{F}^{\#}$ are denoted as $f, g, \ldots$ If $t=\mathrm{f}\left(t_{1}, \ldots, t_{m}\right) \in \mathcal{T}(\mathcal{F}, \mathcal{V})$, we let $t^{\#}$ denote the term $\mathrm{f}^{\#}\left(t_{1}, \ldots, t_{m}\right)$, and we call $t^{\#}$ an $\mathcal{F}^{\#}$-term. An $\mathcal{F}^{\#}$-rule is a rule whose left-hand and right-hand sides are $\mathcal{F}^{\#}$-terms. The set of dependency pairs of $\mathcal{R}$ is

$$
\left\{l^{\#} \rightarrow t^{\#} \mid l \rightarrow r \in \mathcal{R}, t \text { is a subterm of } r, \operatorname{root}(t) \in \mathcal{D}_{\mathcal{R}}\right\} .
$$

A sequence $s_{1} \rightarrow t_{1}, \ldots, s_{n} \rightarrow t_{n}$ of dependency pairs of $\mathcal{R}$ is an $\mathcal{R}$-chain if there exists a substitution $\sigma$ such that $t_{i} \sigma \underset{\mathcal{R}}{\stackrel{*}{\rightarrow}} s_{i+1} \sigma$ holds for every two consecutive pairs $s_{i} \rightarrow t_{i}$ and $s_{i+1} \rightarrow t_{i+1}$ in the sequence.

Theorem 1 ([3]). $\mathcal{R}$ is non-terminating iff there exists an infinite $\mathcal{R}$-chain.

The dependency graph of $\mathcal{R}$ is the graph whose nodes are the dependency pairs of $\mathcal{R}$ and there is an arc from $s \rightarrow t$ to $u \rightarrow v$ iff $s \rightarrow t, u \rightarrow v$ is an $\mathcal{R}$-chain. This graph is not computable in general since it is undecidable whether two dependency pairs of $\mathcal{R}$ form an $\mathcal{R}$-chain. Hence, for automation, one constructs an estimated graph containing all the arcs of the real graph. This is done by computing connectable terms, which form a superset of those terms $s, t$ where $s \sigma \underset{\mathcal{R}}{\stackrel{*}{\rightarrow}} t \sigma$ holds for some substitution $\sigma$. The approximation uses the transformations CAP 
and REN where, for any $t \in \mathcal{T}\left(\mathcal{F} \cup \mathcal{F}^{\#}, \mathcal{V}\right)$, $\operatorname{CAP}(t)$ (resp. REN $\left.(t)\right)$ results from replacing all subterms of $t$ with defined root symbol (resp. all variable occurrences in $t$ ) by different new variables not previously met. More formally:

$$
\begin{aligned}
\operatorname{CAP}(x)= & x \text { if } x \in \mathcal{V} \\
\operatorname{CAP}\left(f\left(t_{1}, \ldots, t_{m}\right)\right)= & \begin{cases}\text { a new variable not previously met if } f \in \mathcal{D}_{\mathcal{R}} \\
f\left(\operatorname{CAP}\left(t_{1}\right), \ldots, \operatorname{CAP}\left(t_{m}\right)\right)\end{cases} \\
\operatorname{REN}(x)= & \text { a new variable not previously met } \notin \mathcal{D}_{\mathcal{R}} \\
& \text { if } x \text { is an occurrence of a variable } \\
\operatorname{REN}\left(f\left(t_{1}, \ldots, t_{m}\right)\right)= & f\left(\operatorname{REN}\left(t_{1}\right), \ldots, \operatorname{REN}\left(t_{m}\right)\right)
\end{aligned}
$$

A term $s$ is connectable to a term $t$ if $\operatorname{REN}(\operatorname{CAP}(s))$ unifies with $t$. An $\mathcal{F}^{\#}$-rule $l \rightarrow r$ is connectable to an $\mathcal{F}^{\#}$-rule $s \rightarrow t$ if $r$ is connectable to $s$. The estimated dependency graph of $\mathcal{R}$ is denoted as $D G(\mathcal{R})$. Its nodes are the dependency pairs of $\mathcal{R}$ and there is an arc from $N$ to $N^{\prime}$ iff $N$ is connectable to $N^{\prime}$. We let $S C C(\mathcal{R})$ denote the set of strongly connected components of $D G(\mathcal{R})$ that contain at least one arc. Hence, a strongly connected component consisting of a unique node is in $S C C(\mathcal{R})$ only if there is an arc from the node to itself.

Example 4. Let $\mathcal{R}$ be the TRS of Ex. 1. We have $S C C(\mathcal{R})=\{\mathcal{C}\}$ where $\mathcal{C}$ consists of the node $N=\mathrm{f}^{\#}(\mathrm{~s}(0), \mathrm{s}(1), x) \rightarrow \mathrm{f}^{\#}(x, x, x)$ and of the $\operatorname{arc}(N, N)$.

Example 5. Let $\mathcal{R}^{\prime}=\{\mathrm{f}(0) \rightarrow \mathrm{f}(1), \mathrm{f}(2) \rightarrow \mathrm{f}(0), 1 \rightarrow 0\}$. We have $S C C\left(\mathcal{R}^{\prime}\right)=$ $\left\{\mathcal{C}^{\prime}\right\}$ where $\mathcal{C}^{\prime}$ consists of the nodes $N_{1}=\mathrm{f}^{\#}(0) \rightarrow \mathrm{f}^{\#}(1)$ and $N_{2}=\mathrm{f}^{\#}(2) \rightarrow \mathrm{f}^{\#}(0)$ and of the $\operatorname{arcs}\left\{N_{1}, N_{2}\right\} \times\left\{N_{1}, N_{2}\right\} \backslash\left\{\left(N_{2}, N_{2}\right)\right\}$. The strongly connected component of $D G\left(\mathcal{R}^{\prime}\right)$ which consists of the unique node $\mathrm{f}^{\#}(0) \rightarrow 1^{\#}$ does not belong to $S C C\left(\mathcal{R}^{\prime}\right)$ because it has no arc.

Finite sequences are written as $\left[e_{1}, \ldots, e_{n}\right]$. We let :: denote the concatenation operator over finite sequences. A path in $D G(\mathcal{R})$ is a finite sequence $\left[N_{1}, N_{2}, \ldots, N_{n}\right]$ of nodes where, for each $1 \leq i<n$, there is an arc from $N_{i}$ to $N_{i+1}$. When there is also an arc from $N_{n}$ to $N_{1}$, the path is called a cycle. It is called a simple cycle if, moreover, there is no repetition of nodes (modulo variable renaming).

\section{Guided unfoldings}

In the sequel of this paper, we let $\mathcal{R}$ denote a TRS over $\mathcal{F} \cup \mathcal{V}$.

While the method sketched in Ex. 2 can be applied directly to the TRS $\mathcal{R}$ under analysis, we use a refinement based on the estimated dependency graph of $\mathcal{R}$. The cycles in $D G(\mathcal{R})$ are over-approximations of the infinite $\mathcal{R}$-chains i.e., any infinite $\mathcal{R}$-chain corresponds to a cycle in the graph but some cycles in the graph may not correspond to any $\mathcal{R}$-chain. Moreover, by Theorem 1 , if we find an infinite $\mathcal{R}$-chain then we have proved that $\mathcal{R}$ is non-terminating. Hence, we concentrate on the cycles in $D G(\mathcal{R})$. We try to solve them, i.e., to find out if 
they correspond to any infinite $\mathcal{R}$-chain. This is done by iteratively unfolding the $\mathcal{F}^{\#}$-rules of the cycles. If the semi-unification test succeeds on one of the generated unfolded rules, then we have found a loop.

Definition 1 (Syntactic loop). A syntactic loop in $\mathcal{R}$ is a finite sequence $\left[N_{1}, \ldots, N_{n}\right]$ of distinct (modulo variable renaming) $\mathcal{F}^{\#}$-rules where, for each $1 \leq i<n, N_{i}$ is connectable to $N_{i+1}$ and $N_{n}$ is connectable to $N_{1}$. We identify syntactic loops consisting of the same (modulo variable renaming) elements, not necessarily in the same order.

Note that the simple cycles in $D G(\mathcal{R})$ are syntactic loops. For any $\mathcal{C} \in$ $S C C(\mathcal{R})$, we let $s$-cycles $(\mathcal{C})$ denote the set of simple cycles in $\mathcal{C}$. We also let

$$
\text { s-cycles }(\mathcal{R})=\cup_{\mathcal{C} \in S C C(\mathcal{R})} s \text {-cycles }(\mathcal{C})
$$

be the set of simple cycles in $\mathcal{R}$. The rules of any simple cycle in $\mathcal{R}$ are assumed to be pairwise variable disjoint.

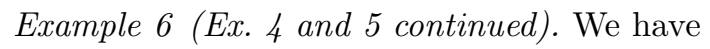

$$
\text { s-cycles }(\mathcal{R})=\{[N]\} \quad \text { and } \quad s \text {-cycles }\left(\mathcal{R}^{\prime}\right)=\left\{\left[N_{1}\right],\left[N_{1}, N_{2}\right]\right\}
$$

with, in s-cycles $\left(\mathcal{R}^{\prime}\right),\left[N_{1}, N_{2}\right]=\left[N_{2}, N_{1}\right]$.

The operators we use for unfolding an $\mathcal{F}^{\#}$-rule $R$ at a disagreement position $p$ are defined as follows. They are guided by a given term $u$ and they only work on the non-variable subterms of $R$. They unify a subterm of $R$ with a subterm of $u$, see (1) in Def. 2-3, This corresponds to what we did in Ex. 2 for generating $V_{1}^{\prime}$ from $V_{1}$, but in the definitions below we do not only consider $p$, we consider all its prefixes. The operators also unfold $R$ using narrowing, see (2) in Def. 2, 33: there, $l^{\prime} \rightarrow r^{\prime} \ll \mathcal{R}$ means that $l^{\prime} \rightarrow r^{\prime}$ is a new occurrence of a rule of $\mathcal{R}$ that contains new variables not previously met. This corresponds to what we did in Ex. 2 for generating $V_{1}$ from $R_{1}$. In contrast to (1), the positions that are greater than $p$ are also considered in (2); for instance in Ex. 2, we unfolded the inner subterm 0 of the disagreement pair component $s(0)$.

Definition 2 (Forward guided unfoldings). Let $l \rightarrow r$ be an $\mathcal{F}^{\#}$-rule, $s$ be an $\mathcal{F}^{\#}$-term and $p \in D \operatorname{Pos}(r, s)$. The forward unfoldings of $l \rightarrow r$ at position $p$, guided by $s$ and w.r.t. $\mathcal{R}$ are

$$
\begin{aligned}
F_{\mathcal{R}}(l \rightarrow r, s, p)= & \left\{\begin{array}{l}
U \mid \begin{array}{l}
q \in \operatorname{NPos}(r, p), q \leq p \\
\theta=\operatorname{mgu}\left(\left.r\right|_{q},\left.s\right|_{q}\right), U=(l \rightarrow r) \theta
\end{array}
\end{array}\right\}^{(1)} \cup \\
& \left\{\begin{array}{ll}
U \mid \begin{array}{l}
q \in \operatorname{NPos}(r, p), l^{\prime} \rightarrow r^{\prime} \ll \mathcal{R} \\
\theta=\operatorname{mgu}\left(\left.r\right|_{q}, l^{\prime}\right), U=\left(l \rightarrow r\left[q \leftarrow r^{\prime}\right]\right) \theta
\end{array}
\end{array}\right\}^{(2)} .
\end{aligned}
$$

Definition 3 (Backward guided unfoldings). Let $s \rightarrow t$ be an $\mathcal{F}^{\#}$-rule, $r$ be an $\mathcal{F}^{\#}$-term and $p \in \operatorname{DPos}(r, s)$. The backward unfoldings of $s \rightarrow t$ at position $p$, 
guided by $r$ and w.r.t. $\mathcal{R}$ are

$$
\begin{aligned}
B_{\mathcal{R}}(s \rightarrow t, r, p)= & \left\{\begin{array}{l}
U \in \operatorname{NPos}(s, p), q \leq p \\
\theta=\operatorname{mgu}\left(\left.r\right|_{q},\left.s\right|_{q}\right), U=(s \rightarrow t) \theta
\end{array}\right\}^{(1)} \cup \\
& \left\{\begin{array}{l}
U \mid \begin{array}{l}
q \in \operatorname{NPos}(s, p), l^{\prime} \rightarrow r^{\prime} \ll \mathcal{R} \\
\theta=\operatorname{mgu}\left(\left.s\right|_{q}, r^{\prime}\right), U=\left(s\left[q \leftarrow l^{\prime}\right] \rightarrow t\right) \theta
\end{array}
\end{array}\right\}^{(2)} .
\end{aligned}
$$

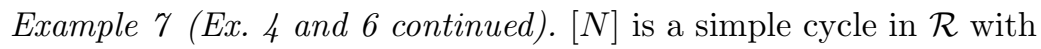

$$
N=\underbrace{\mathrm{f}^{\#}(\mathrm{~s}(0), \mathrm{s}(1), x)}_{s} \rightarrow \underbrace{\mathrm{f}^{\#}(x, x, x)}_{t} .
$$

Let $r=t$. Then $p=1 \in \operatorname{DPos}(r, s)$. Moreover, $q=1.1 \in N \operatorname{Pos}(s, p)$ because $p \leq q$ and $\left.s\right|_{q}=0$ is not a variable. Let $l^{\prime} \rightarrow r^{\prime}=\mathrm{h} \rightarrow 0 \in \mathcal{R}$. We have $i d=$ $m g u\left(\left.s\right|_{q}, r^{\prime}\right)$. Hence, by (2) in Def. 3, we have

$$
U_{1}=\underbrace{\mathrm{f}^{\#}(\mathrm{~s}(\mathrm{~h}), \mathrm{s}(1), x)}_{s_{1}} \rightarrow \underbrace{\mathrm{f}^{\#}(x, x, x)}_{t_{1}} \in B_{\mathcal{R}}(N, r, p) .
$$

Let $r_{1}=t_{1}$. Then, $p=1 \in \operatorname{DPos}\left(r_{1}, s_{1}\right)$. Moreover, $p \in N \operatorname{Pos}\left(s_{1}, p\right)$ with $\left.s_{1}\right|_{p}=\mathrm{s}(\mathrm{h}), p \leq p$ and $\left.r_{1}\right|_{p}=x$. As $\{x / \mathrm{s}(\mathrm{h})\}=m g u\left(\left.r_{1}\right|_{p},\left.s_{1}\right|_{p}\right)$, by (1) in Def. 3 we have

$$
U_{1}^{\prime}=\underbrace{\mathrm{f} \#(\mathrm{~s}(\mathrm{~h}), \mathrm{s}(1), \mathrm{s}(\mathrm{h}))}_{s_{1}^{\prime}} \rightarrow \underbrace{\mathrm{f}^{\#}(\mathrm{~s}(\mathrm{~h}), \mathrm{s}(\mathrm{h}), \mathrm{s}(\mathrm{h}))}_{t_{1}^{\prime}} \in B_{\mathcal{R}}\left(U_{1}, r_{1}, p\right) .
$$

Let $r_{1}^{\prime}=t_{1}^{\prime}$. Then, $p^{\prime}=2.1 \in \operatorname{DPos}\left(r_{1}^{\prime}, s_{1}^{\prime}\right)$ with $p^{\prime} \in N \operatorname{Pos}\left(s_{1}^{\prime}, p^{\prime}\right)$. Let $l^{\prime \prime} \rightarrow r^{\prime \prime}=$ $\mathrm{h} \rightarrow 1 \in \mathcal{R}$. We have $i d=m g u\left(\left.s_{1}^{\prime}\right|_{p^{\prime}}, r^{\prime \prime}\right)$. Hence, by (2) in Def. 3, we have

$$
U_{1}^{\prime \prime}=\mathrm{f}^{\#}(\mathrm{~s}(\mathrm{~h}), \mathrm{s}(\mathrm{h}), \mathrm{s}(\mathrm{h})) \rightarrow \mathrm{f}^{\#}(\mathrm{~s}(\mathrm{~h}), \mathrm{s}(\mathrm{h}), \mathrm{s}(\mathrm{h})) \in B_{\mathcal{R}}\left(U_{1}^{\prime}, r_{1}^{\prime}, p^{\prime}\right) .
$$

Our approach consists of iteratively unfolding syntactic loops using the following operator.

Definition 4 (Guided unfoldings). Let $X$ be a set of syntactic loops of $\mathcal{R}$. The guided unfoldings of $X$ w.r.t. $\mathcal{R}$ are defined as

$$
\begin{aligned}
& G U_{\mathcal{R}}(X)=\left\{\begin{array}{l|l}
L::[U]:: L^{\prime} & \begin{array}{l}
L::[l \rightarrow r, s \rightarrow t]:: L^{\prime} \in X, \theta=\operatorname{mgu}(r, s) \\
U=(l \rightarrow t) \theta, L::[U]:: L^{\prime} \text { is a syntactic loop }
\end{array}
\end{array}\right\} \\
& \left\{\begin{array}{l|l}
L::[U, s \rightarrow t]:: L^{\prime} & \begin{array}{l}
L::[l \rightarrow r, s \rightarrow t]:: L^{\prime} \in X \\
p \in D P o s(r, s), U \in F_{\mathcal{R}}(l \rightarrow r, s, p) \\
L::[U, s \rightarrow t]:: L^{\prime} \text { is a syntactic loop }
\end{array}
\end{array}\right\} \quad U \\
& \left\{\begin{array}{l|l}
L::[l \rightarrow r, U]:: L^{\prime} & \begin{array}{l}
L::[l \rightarrow r, s \rightarrow t]:: L^{\prime} \in X \\
p \in D P o s(r, s), U \in B_{\mathcal{R}}(s \rightarrow t, r, p) \\
L::[l \rightarrow r, U]:: L^{\prime} \text { is a syntactic loop }
\end{array}
\end{array}\right\} \cup \\
& \left\{\begin{array}{l|l}
{[U]} & \begin{array}{l}
{[l \rightarrow r] \in X, p \in \operatorname{DPos}(r, l)} \\
U \in F_{\mathcal{R}}(l \rightarrow r, l, p) \cup B_{\mathcal{R}}(l \rightarrow r, r, p) \\
{[U] \text { is a syntactic loop }}
\end{array}
\end{array}\right\} .
\end{aligned}
$$


The general idea is to compress the syntactic loops into singletons by iterated applications of this operator. The semi-unification criterion can then be applied to these singletons, see Theorem 2 below. Compression takes place in case (1) of Def. 4 when the right-hand side of a rule unifies with the left-hand side of its successor, then both rules are merged. When merging two successive rules is not possible yet, the operators $F_{\mathcal{R}}$ and $B_{\mathcal{R}}$ are applied to try to transform the rules into mergeable ones, see cases (2) and (3). Once a syntactic loop has been compressed to a singleton, we keep on unfolding (case (4)) to try reaching a compressed form satisfying the semi-unification criterion. Note that after an unfolding step, we might get a sequence which is not a syntactic loop: the newly generated rule $U$ might be identical to another rule in the sequence or it might not be connectable to its predecessor or successor. So, (1)-(4) require that the generated sequence is a syntactic loop.

The guided unfolding semantics is defined as follows, in the style of [113].

Definition 5 (Guided unfolding semantics). The guided unfolding seman-

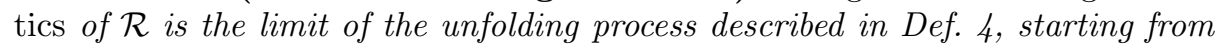
the simple cycles in $\mathcal{R}$ : $\operatorname{gunf}(\mathcal{R})=\cup_{n \in \mathbb{N}} \operatorname{gunf}(\mathcal{R}, n)$ where, for all $n \in \mathbb{N}$,

$$
\operatorname{gunf}(\mathcal{R}, n)=\left(G U_{\mathcal{R}} \uparrow n\right)(s \text {-cycles }(\mathcal{R})) .
$$

This semantics is very similar to the overlap closure [7] of $\mathcal{R}$ (denoted by $O C(\mathcal{R}))$. A difference is that for computing $\operatorname{gunf}(\mathcal{R})$ one starts from dependency pairs of $\mathcal{R}(s$-cycles $(\mathcal{R}))$, whereas for computing $O C(\mathcal{R})$ one starts directly from

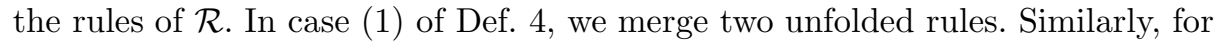
computing $O C(\mathcal{R})$ one overlaps closures with closures. However, in cases (2)-(4) the operators $F_{\mathcal{R}}$ and $B_{\mathcal{R}}$ narrow an unfolded rule with a rule of $\mathcal{R}$, not with another unfolded rule, unlike in the computation of $O C(\mathcal{R})$.

Example 8. By Ex. 7 and (4) in Def. 4, we have $\left[U_{1}^{\prime \prime}\right] \in \operatorname{gunf}(\mathcal{R}, 3)$.

Example 9. Let $\mathcal{R}=\{\mathrm{f}(0) \rightarrow \mathrm{g}(1), \mathrm{g}(1) \rightarrow \mathrm{f}(0)\}$. Then, $S C C(\mathcal{R})=\{\mathcal{C}\}$ where $\mathcal{C}$ consists of the nodes $N_{1}=\mathrm{f}^{\#}(0) \rightarrow \mathrm{g}^{\#}(1)$ and $N_{2}=\mathrm{g}^{\#}(1) \rightarrow \mathrm{f}^{\#}(0)$ and of the $\operatorname{arcs}\left(N_{1}, N_{2}\right)$ and $\left(N_{2}, N_{1}\right)$. Moreover, s-cycles $(\mathcal{R})=\left\{\left[N_{1}, N_{2}\right]\right\}$. As $i d=$ $m g u\left(\mathrm{~g}^{\#}(1), \mathrm{g}^{\#}(1)\right)$ and $\left(\mathrm{f}^{\#}(0) \rightarrow \mathrm{f}^{\#}(0)\right) i d=\mathrm{f}^{\#}(0) \rightarrow \mathrm{f}^{\#}(0)$, by (1) in Def. 目 we have $\left[\mathrm{f}^{\#}(0) \rightarrow \mathrm{f} \#(0)\right] \in \operatorname{gunf}(\mathcal{R}, 1)$.

Proposition 1. For any $n \in \mathbb{N}$ and $\left[s^{\#} \rightarrow t^{\#}\right] \in \operatorname{gunf}(\mathcal{R}, n)$ there exists some context $C$ such that $s \underset{\mathcal{R}}{\stackrel{+}{\rightarrow}} C[t]$.

Proof. For some context $C$, we have $s \rightarrow C[t] \in \operatorname{unf}(\mathcal{R})$ where $\operatorname{unf}(\mathcal{R})$ is the unfolding semantics defined in [13]. So, by Prop. 3.12 of [13], $\underset{\mathcal{R}}{\stackrel{+}{\rightarrow}} C[t]$.

\section{Inferring terms that loop}

As in [13, we use semi-unification [10] for detecting loops. Semi-unification encompasses both matching and unification, and a polynomial-time algorithm for it can be found in [8]. 
Theorem 2. For any $n \in \mathbb{N}$, if there exist $\left[s^{\#} \rightarrow t^{\#}\right] \in \operatorname{gunf}(\mathcal{R}, n)$ and some substitutions $\theta_{1}$ and $\theta_{2}$ such that $s \theta_{1} \theta_{2}=t \theta_{1}$, then the term $s \theta_{1}$ loops w.r.t. $\mathcal{R}$.

Proof. By Prop. 团 $s \underset{\mathcal{R}}{\stackrel{+}{\rightarrow}} C[t]$ for some context $C$. Since $\underset{\mathcal{R}}{\rightarrow}$ is stable, we have

$$
s \theta_{1} \underset{\mathcal{R}}{\stackrel{+}{\rightarrow}} C[t] \theta_{1} \quad \text { i.e., } \quad s \theta_{1} \underset{\mathcal{R}}{\stackrel{+}{\rightarrow}} C \theta_{1}\left[t \theta_{1}\right] \quad \text { i.e., } \quad s \theta_{1} \underset{\mathcal{R}}{\stackrel{+}{\rightarrow}} C \theta_{1}\left[s \theta_{1} \theta_{2}\right] .
$$

Hence, $s \theta_{1}$ loops w.r.t. $\mathcal{R}$.

Example 10 (Ex. 8 continued). We have

$$
[\underbrace{\mathrm{f} \#(\mathrm{~s}(\mathrm{~h}), \mathrm{s}(\mathrm{h}), \mathrm{s}(\mathrm{h})) \rightarrow \mathrm{f}^{\#}(\mathrm{~s}(\mathrm{~h}), \mathrm{s}(\mathrm{h}), \mathrm{s}(\mathrm{h}))}_{U_{1}^{\prime \prime}}] \in \operatorname{gunf}(\mathcal{R}, 3)
$$

with $\mathrm{f}(\mathrm{s}(\mathrm{h}), \mathrm{s}(\mathrm{h}), \mathrm{s}(\mathrm{h})) \theta_{1} \theta_{2}=\mathrm{f}(\mathrm{s}(\mathrm{h}), \mathrm{s}(\mathrm{h}), \mathrm{s}(\mathrm{h})) \theta_{1}$ for $\theta_{1}=\theta_{2}=i d$. Consequently, $\mathrm{f}(\mathrm{s}(\mathrm{h}), \mathrm{s}(\mathrm{h}), \mathrm{s}(\mathrm{h})) \theta_{1}=\mathrm{f}(\mathrm{s}(\mathrm{h}), \mathrm{s}(\mathrm{h}), \mathrm{s}(\mathrm{h}))$ loops w.r.t. $\mathcal{R}$.

Example 11 (Ex. 9 continued). [f\# $\left.(0) \rightarrow \mathrm{f}^{\#}(0)\right] \in \operatorname{gunf}(\mathcal{R}, 1)$ with $\mathrm{f}(0) \theta_{1} \theta_{2}=$ $\mathrm{f}(0) \theta_{1}$ for $\theta_{1}=\theta_{2}=i d$. Hence, $\mathrm{f}(0) \theta_{1}=\mathrm{f}(0)$ loops w.r.t. $\mathcal{R}$.

The substitutions $\theta_{1}$ and $\theta_{2}$ that we use in the next example are more sophisticated than in Ex. 10 and Ex. 11.

Example 12. Let $\mathcal{R}=\{\mathrm{f}(\mathrm{g}(x, 0), y) \rightarrow \mathrm{f}(\mathrm{g}(0, x), \mathrm{h}(y))\}$. Then, $S C C(\mathcal{R})=\{\mathcal{C}\}$ where $\mathcal{C}$ consists of the node $N=\mathrm{f}^{\#}(\mathrm{~g}(x, 0), y) \rightarrow \mathrm{f}^{\#}(\mathrm{~g}(0, x), \mathrm{h}(y))$ and of the $\operatorname{arc}(N, N)$. Moreover, s-cycles $(\mathcal{R})=\{[N]\}$ hence $[N] \in \operatorname{gunf}(\mathcal{R}, 0)$. Therefore, as $\mathrm{f}(\mathrm{g}(x, 0), y) \theta_{1} \theta_{2}=\mathrm{f}(\mathrm{g}(0, x), \mathrm{h}(y)) \theta_{1}$ for $\theta_{1}=\{x / 0\}$ and $\theta_{2}=\{y / \mathrm{h}(y)\}$, by Theorem 2 we have that $\mathrm{f}(\mathrm{g}(x, 0), y) \theta_{1}=\mathrm{f}(\mathrm{g}(0,0), y)$ loops w.r.t. $\mathcal{R}$.

We do not have an example where semi-unification is necessary for detecting a loop. In every example that we have considered, matching or unification were enough. However, semi-unification sometimes allows us to detect loops earlier in the unfolding process than with matching and unification. This is important in practice, because the number of unfolded rules can grow rapidly from iteration to iteration.

Example 13 (Ex. 12 continued). Semi-unification allows us to detect a loop at iteration 0 of $G U_{\mathcal{R}}$. But a loop can also be detected at iteration 1 using matching. Indeed, we have

$$
N=\underbrace{\mathrm{f} \#(\mathrm{~g}(x, 0), y)}_{l} \rightarrow \underbrace{\mathrm{f}^{\#}(\mathrm{~g}(0, x), \mathrm{h}(y))}_{r}
$$

with $p=1.1 \in \operatorname{DPos}(r, l)$. Hence,

$$
U=\underbrace{\mathrm{f}^{\#}(\mathrm{~g}(0,0), y)}_{s^{\#}} \rightarrow \underbrace{\mathrm{f}^{\#}(\mathrm{~g}(0,0), \mathrm{h}(y))}_{t^{\#}} \in F_{\mathcal{R}}(l \rightarrow r, l, p) .
$$

So, by (4) in Def. 4. we have $[U] \in \operatorname{gunf}(\mathcal{R}, 1)$. Notice that $s \theta=t$ for $\theta=$ $\{y / \mathrm{h}(y)\}$, so $s$ matches $t$. Moreover, by Theorem 2, $s=\mathrm{f}(\mathrm{g}(0,0), y)$ loops w.r.t. $\mathcal{R}\left(\right.$ take $\theta_{1}=i d$ and $\left.\theta_{2}=\theta\right)$. 


\section{Further comparisons with the approach of [13]}

The approach that we have presented in [13] relies on an unfolding operator $U_{\mathcal{R}}$ (where $\mathcal{R}$ is the TRS under analysis) which is also based on forward and backward narrowing (as $F_{\mathcal{R}}$ and $B_{\mathcal{R}}$ herein). But, unlike the technique that we have presented above, it directly unfolds the rules (not the dependency pairs) of $\mathcal{R}$ and it does not compute any SCC. Moreover, it consists of a thorough computation of the iterations of $U_{\mathcal{R}}$ followed by a mechanism for eliminating rules that cannot be further unfolded to a rule $l \rightarrow r$ where $l$ semi-unifies with $r$. Such rules are said to be root-useless. The set of root-useless rules is an overapproximation of the set of useless rules (rules that cannot contribute to detecting a loop), hence the elimination technique of [13] may remove some rules which are actually useful for detecting a loop. Our non-termination analyser which is based on 13 uses a time limit. It stops whenever it has detected a loop within the limit (then it answers NO, standing for No, this TRS does not terminate as in the Termination Competition [15]) or when the limit has been reached (then it answers TIME OUT) or when no more unfolded rule could be generated at some point within the limit $\left(\left(U_{\mathcal{R}} \uparrow n\right)(\mathcal{R})=\emptyset\right.$ for some $\left.n\right)$. In the last situation, either the TRS under analysis is not looping (it is terminating or non-looping non-terminating) or it is looping but a loop for it cannot be captured by the approach (for instance, the elimination mechanism has removed all the useful rules). In such a situation, our analyser answers DON'T KNOW.

Example 14. Consider the terminating TRS $\mathcal{R}=\{0 \rightarrow 1\}$. As the left-hand (resp. right-hand) side of the rule of $\mathcal{R}$ cannot be narrowed backwards (resp. forwards) with $\mathcal{R}$ then we have $\left(U_{\mathcal{R}} \uparrow 1\right)(\mathcal{R})=\emptyset$.

In contrast, the approach that we have presented in Sect. 34 above avoids the generation of some rules by only unfolding disagreement pairs. Currently, in terms of loop detection power, we do not have any theoretical comparison between this new technique and that of [13. Our new non-termination analyser also uses a time limit and answers NO, TIME OUT or DON'T KNOW when no more unfolded rules are generated at some point $\operatorname{gunf}(\mathcal{R}, n)=\emptyset$ for some $n$, as in Ex. (14). Moreover, it allows the user to fix a selection strategy of disagreement pairs: in Def. 4, the conditions $p \in D P o s(r, s)$ (cases (2)-(3)) and $p \in D P o s(r, l)$ (case (4)) are replaced with $p \in \operatorname{select}_{\mathcal{R}}(l \rightarrow r, s \rightarrow t)$ and $p \in \operatorname{select}_{\mathcal{R}}(l \rightarrow r, l \rightarrow r)$ respectively, where select $_{\mathcal{R}}$ can be one of the following functions.

Selection of all the pairs: select_all $_{\mathcal{R}}(l \rightarrow r, s \rightarrow t)=\operatorname{DPos}(r, s)$.

Leftmost selection: if $D P o s(r, s)=\emptyset$ then select_lm $_{\mathcal{R}}(l \rightarrow r, s \rightarrow t)=\emptyset$, otherwise select_l $m_{\mathcal{R}}(l \rightarrow r, s \rightarrow t)=\{p\}$ where $p$ is the leftmost disagreement position of $r$ and $s$.

\section{Leftmost selection with non-empty unfoldings:}

$$
\text { select_lmne }_{\mathcal{R}}(l \rightarrow r, s \rightarrow t)=\{p\}
$$

where $p$ is the leftmost disagreement position of $r$ and $s$ such that

$$
F_{\mathcal{R}}(l \rightarrow r, s, p) \cup B_{\mathcal{R}}(s \rightarrow t, r, p) \neq \emptyset .
$$


If such a position $p$ does not exist then select_lmne $e_{\mathcal{R}}(l \rightarrow r, s \rightarrow t)=\emptyset$.

Example 15. Let $\mathcal{R}=\{\mathrm{f}(\mathrm{s}(0), \mathrm{s}(1), z) \rightarrow \mathrm{f}(x, y, z)\}$ and $l=\mathrm{f} \#(\mathrm{~s}(0), \mathrm{s}(1), z)$ and $r=\mathrm{f}^{\#}(x, y, z)$. Then, we have select_all $\mathcal{R}(l \rightarrow r, l \rightarrow r)=\operatorname{DPos}(r, l)=\{1,2\}$. Moreover, 1 is the leftmost disagreement position of $r$ and $l$ because $\left.r\right|_{1}=x$ occurs to the left of $\left.r\right|_{2}=y$ in $r$ and $\left.l\right|_{1}=\mathrm{s}(0)$ occurs to the left of $\left.l\right|_{2}=\mathrm{s}(1)$ in $l$. Therefore, we have select_lm $m_{\mathcal{R}}(l \rightarrow r, l \rightarrow r)=\{1\}$.

Example 16. Let $\mathcal{R}=\{\mathrm{f}(x, x) \rightarrow \mathrm{f}(\mathrm{g}(x), \mathrm{h}(x)), \mathrm{h}(x) \rightarrow \mathrm{g}(x)\}$. Then, $S C C(\mathcal{R})=$ $\{\mathcal{C}\}$ where $\mathcal{C}$ consists of the node $N=l \rightarrow r=\mathrm{f}^{\#}(x, x) \rightarrow \mathrm{f}^{\#}(\mathrm{~g}(x), \mathrm{h}(x))$ and of the $\operatorname{arc}(N, N)$. Then, $\operatorname{DPos}(r, l)=\{1,2\}$ and $\operatorname{select}_{-} \operatorname{lm}_{\mathcal{R}}(N, N)=\{1\}$. As $F_{\mathcal{R}}(N, l, 1) \cup B_{\mathcal{R}}(N, r, 1)=\emptyset$ and $F_{\mathcal{R}}(N, l, 2) \cup B_{\mathcal{R}}(N, r, 2) \neq \emptyset$ (for instance, $\mathrm{f}^{\#}(x, x) \rightarrow \mathrm{f}^{\#}(\mathrm{~g}(x), \mathrm{g}(x)) \in F_{\mathcal{R}}(N, l, 2)$ is obtained from narrowing $\left.r\right|_{2}=\mathrm{h}(x)$ forwards with $\mathrm{h}(x) \rightarrow \mathrm{g}(x))$, then select_lmne $\mathcal{R}(N, N)=\{2\}$.

As the approach of [13], and depending on the strategy used for selecting disagreement pairs, our new technique is not complete in the sense that it may miss some loop witnesses.

Example 17 (Ex. 16] continued). We have $\operatorname{gunf}(\mathcal{R}, 0)=s$-cycles $(\mathcal{R})=\{[N]\}$. As $l$ does not semi-unify with $r$, no loop is detected from $\operatorname{gunf}(\mathcal{R}, 0)$, so we go on and compute $\operatorname{gunf}(\mathcal{R}, 1)$. Only case $(4)$ of Def. 4 is applicable to $[N]$. First, suppose that select $\mathcal{R}_{\mathcal{R}}=$ select_lm $_{\mathcal{R}}$. Then, $\operatorname{select}_{\mathcal{R}}(N, N)=\{1\}$ and, as $F_{\mathcal{R}}(N, l, 1) \cup B_{\mathcal{R}}(N, r, 1)=\emptyset$, case (4) does not produce any rule. Consequently, we have $\operatorname{gunf}(\mathcal{R}, 1)=\emptyset$, hence no loop is detected for $\mathcal{R}$. Now, suppose that select $_{\mathcal{R}}=$ select_lmne $_{\mathcal{R}}$. Then, select se $_{\mathcal{R}}(N, N)=\{2\}$. Narrowing $\left.r\right|_{2}=\mathrm{h}(x)$ forwards with $\mathrm{h}(x) \rightarrow \mathrm{g}(x)$, we get the rule $N^{\prime}=\mathrm{f}^{\#}(x, x) \rightarrow \mathrm{f}^{\#}(\mathrm{~g}(x), \mathrm{g}(x))$ which is an element of $F_{\mathcal{R}}(N, l, 2)$. Hence, $\left[N^{\prime}\right] \in \operatorname{gunf}(\mathcal{R}, 1)$. As in $N^{\prime}$ we have that $\mathrm{f}(x, x)$ semi-unifies with $\mathrm{f}(\mathrm{g}(x), \mathrm{g}(x))$ (take $\theta_{1}=i d$ and $\left.\theta_{2}=\{x / \mathrm{g}(x)\}\right)$, then $\mathrm{f}(x, x)$ loops w.r.t. $\mathcal{R}$. This loop is also detected by the approach of [13].

\section{Experiments}

We have implemented the technique of this paper in our analyser NT1 (NonTermination Inference). For our experiments, we have extracted from the directory TRS_Standard of the TPBD [17 all the valid rewrite systems 2 that were either proved looping or unproved 3 during the Termination Competition 2017 (TC'17) [15]. Otherwise stated, we removed from TRS_Standard all the nonvalid TRSs and all the TRSs that were proved terminating or non-looping nonterminating by a tool participating in the competition. We ended up with a set $\mathcal{S}$ of 333 rewrite systems. We let $\mathcal{L}$ (resp. $\mathcal{U}$ ) be the subset of $\mathcal{S}$ consisting of all

1 http://lim.univ-reunion.fr/staff/epayet/Research/NTI/NTI.html

2 Surprisingly, the subdirectory Transformed_CSR_04 contains 60 files where a pair $l \rightarrow r$ with $\operatorname{Var}(r) \nsubseteq \operatorname{Var}(l)$ occurs. These pairs are not valid rewrite rules.

${ }^{3}$ By unproved we mean that no tool succeeded in proving that these TRSs were terminating or non-terminating (all the tools failed on these TRSs). 
the systems that were proved looping (resp. that were unproved) during TC'17. Some characteristics of $\mathcal{L}$ and $\mathcal{U}$ are reported in Table 1 . Note that the complete set of simple cycles of a TRS may be really huge, hence NTI only computes a subset of it. The simple cycle characteristics given in Table1 1 relate to the subsets computed by NTI.

\begin{tabular}{|c|c|c|c|c|c|c|}
\hline \multirow{2}{*}{$\mathcal{S}=\mathcal{L} \uplus \mathcal{U}(333$ TRSs $)$} & \multicolumn{3}{|c|}{$\mathcal{L}$ (173 TRSs) } & \multicolumn{3}{|c|}{$\mathcal{U}(160 \mathrm{TRSs})$} \\
\hline & Min & $\operatorname{Max}$ & Average & Min & $\operatorname{Max}$ & Average \\
\hline TRS size & $\begin{array}{|ll|}1 & {[17]} \\
\end{array}$ & $104[1]$ & 11.08 & & $837[1]$ & 64.78 \\
\hline Number of SCCs & $1[101]$ & $12[1]$ & 1.95 & $\begin{array}{ll}1 & {[70]} \\
\end{array}$ & $130[1]$ & 6.14 \\
\hline SCC size & \begin{tabular}{|ll} 
& {$[96]$} \\
\end{tabular} & $192[1]$ & 4.44 & \begin{tabular}{|ll}
1 & {$[54]$} \\
\end{tabular} & $473[1]$ & 10.79 \\
\hline Number of simple cycles & [47] & $185[1]$ & 8.55 & $\overline{[15]}$ & $\overline{1,176[1]}$ & $\overline{69.02}$ \\
\hline $\begin{array}{c}\text { Simple cycle size } \\
\end{array}$ & $1[157]$ & $9[2]$ & 2.23 & $1[157]$ & $9[4]$ & 2.21 \\
\hline Number of symbols & $\begin{array}{|ll|}1 & {[4]} \\
\end{array}$ & $66[1]$ & 9.09 & \begin{tabular}{|ll}
2 & {$[7]$} \\
\end{tabular} & $259[1]$ & 24.14 \\
\hline Symbol arity & $0[153]$ & $5[2]$ & 1.07 & $0[150]$ & $12[2]$ & 1.94 \\
\hline Number of defined symbols & 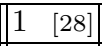 & $58[1]$ & 5.21 & \begin{tabular}{|ll}
1 & {$[15]$} \\
\end{tabular} & $132[2]$ & 17.19 \\
\hline Defined symbol arity & {$[74]$} & $5[2]$ & 1.38 & {$[28]$} & $12[2]$ & 2.27 \\
\hline
\end{tabular}

Table 1. Some characteristics of the analysed TRSs. Sizes are in number of rules. In square brackets, we report the number of TRSs with the corresponding min or max.

We have run our new approach (NTI'18) and that of [13] (NTI'08) on the TRSs of $\mathcal{S}$. The results are reported in Table 2 and Table 3 . We used an Intel 2-core i5 at $2 \mathrm{GHz}$ with $8 \mathrm{~GB}$ of RAM and the time limit fixed for a proof was 120 s. For every selection strategy, NTI'18 issues more successful proofs (NO) and generates less unfolded rules than NTI'08. Moreover, as it avoids the generation of some rules instead of computing all the unfolding and then eliminating some rules (as NTI'08 does), its times are better. At the bottom of the tables, we give the numbers of TRSs proved looping by both approaches and by one approach only. NTI'18 succeeds on all the TRSs of $\mathcal{L}$ on which NTI'08 succeeds, but it fails on one TRS of $\mathcal{U}$ on which NTI'08 succeeds. This is due to our simplified computation of the set of simple cycles: our algorithm does not generate the cycle that would allow NTI'18 to succeed and NTI'18 times out, trying to unfold syntactic loops from which it cannot detect anything. Another point to note is that the implementation of the new approach does not need to run several analyses in parallel to achieve the results presented in Table 2 and Table 3 . One single thread of computation is enough. On the contrary, for the approach of [13, 3 parallel threads are necessary: one with forward unfoldings only, one with backward unfoldings only and one with forward and backward unfoldings together. The results get worse if NTI'08 only runs one thread performing forward and backward unfoldings together. In Table 2 and Table 3 , we report in square brackets the number of successes of NTI'08 when it only runs one thread performing both forward and backward unfoldings. 


\begin{tabular}{|c||r||r|r|r|}
\hline \multirow{2}{*}{$\mathcal{L}$ (173 TRSs) } & \multirow{2}{*}{ NTI'08 } & \multicolumn{3}{|c|}{ NTI'18 } \\
\cline { 3 - 5 } & & select_all & select_lm & select_lmne \\
\hline \hline NO & $152[149]$ & 157 & 157 & 158 \\
\hline DON'T KNOW & 0 & 0 & 1 & 0 \\
\hline TIME OUT & 21 & 16 & 15 & 15 \\
\hline \hline Time & $2,966 \mathrm{~s}$ & $2,194 \mathrm{~s}$ & $1,890 \mathrm{~s}$ & $1,889 \mathrm{~s}$ \\
\hline Generated rules & $11,167,976$ & $9,030,962$ & $8,857,421$ & $8,860,560$ \\
\hline \hline $\mathbf{N O}($ NTI'08) $\cap \mathbf{N O}($ NTI'18) & 152 & 151 & 152 \\
\hline $\mathbf{N O}$ (NTI'08) $\backslash \mathbf{N O}$ (NTI'18) & 0 & 1 & 0 \\
\hline $\mathbf{N O}$ (NTI'18) $\backslash \mathbf{N O}$ (NTI'08) & 5 & 6 & 6 \\
\hline
\end{tabular}

Table 2. Analysis results on the TRSs of $\mathcal{L}$.

\begin{tabular}{|c||r||r|r|r|}
\hline \multirow{2}{*}{$\mathcal{U}(160$ TRSs $)$} & \multirow{2}{*}{ NTI'08 } & \multicolumn{3}{|c|}{ NTI'18 } \\
\cline { 3 - 5 } & & select_all & select_lm & select_lmne \\
\hline \hline NO & $4[3]$ & 6 & 6 & 6 \\
\hline DON'T KNOW & 0 & 0 & 1 & 0 \\
\hline TIME OUT & 156 & 154 & 153 & 154 \\
\hline \hline Time & $18,742 \mathrm{~s}$ & $18,563 \mathrm{~s}$ & $18,414 \mathrm{~s}$ & $18,534 \mathrm{~s}$ \\
\hline Generated rules & $64,011,002$ & $53,134,334$ & $61,245,705$ & $63,300,604$ \\
\hline \hline NO(NTI'08) $\cap \mathbf{N O}$ (NTI'18) & 3 & 3 & 3 \\
\hline NO(NTI'08) $\backslash \mathbf{N O}$ (NTI'18) & 1 & 1 & 1 \\
\hline NO(NTI'18) $\backslash \mathbf{N O}$ (NTI'08) & 3 & 3 & 3 \\
\hline
\end{tabular}

Table 3. Analysis results on the TRSs of $\mathcal{U}$.

Four tools participated in the category TRS Standard of TC'17: AProVE 2]5, MU-TERM [11], NaTT [12] and WANDA [9]. The numbers of TRSs proved looping by each of them during the competition is reported in Table 4 An important point to note here is that the time limit fixed in TC' 17 was 300 s, whereas in our experiments with NTI'18 and NTI'08 it was 120s. Moreover, the machine we used (an Intel 2-core i5 at $2 \mathrm{GHz}$ with $8 \mathrm{~GB}$ of RAM) is much less powerful than the machine used during TC'17 (the StarExec platform [14 running on an Intel Xeon E5-2609 at $2.4 \mathrm{GHz}$ with $129 \mathrm{~GB}$ of RAM). All the tools of TC'17 failed on all the rewrite systems of $\mathcal{U}$. In contrast, NTI'18 (resp. NTI'08) finds a loop for 6 (resp. 4) of them. Regarding $\mathcal{L}$, AProVE was able to prove loopingness of 172 out of 173 TRSs. The only TRS of $\mathcal{L}$ on which AProVE failed $\sqrt{4}$ was proved looping by NaTT. In comparison, our approach succeeds on 158 systems of $\mathcal{L}$, less than AProVE but more than the other tools of TC'17. Similarly to our approach, AProVE handles the SCCs of the estimated dependency graph independently, but it first performs a termination analysis. The non-termination analysis is then only applied to those SCCs that could not be proved terminating. On the

\footnotetext{
${ }^{4}$ Ex6_15_AEL02_FR.xml in the directory TRS_Standard/Transformed_CSR_04
} 
contrary, NTI only performs non-termination proofs. If an SCC is terminating, it cannot prove it and keeps on trying a non-termination proof, unnecessarily generating unfolded rules at the expense of the analysis of the other SCCs. The loop detection techniques implemented in AProVE and NTI'18 are based on the idea of searching for loops by forward and backward narrowing of dependency pairs and by using semi-unification to detect potential loops. This idea has been presented in [6] where heuristics are used to select forward or backward narrowing. Note that in constrast, the technique that we present herein does not use any heuristics and proceeds both forwards and backwards.

\begin{tabular}{|c|c|c|c|c|c|c|}
\hline \multirow{2}{*}{$\mathcal{S}=\mathcal{L} \uplus \mathcal{U}(333$ TRSs $)$} & \multicolumn{4}{|c|}{ TC'17 (time limit $=300 \mathrm{~s})$} & \multicolumn{2}{|c|}{$($ time limit $=120 \mathrm{~s})$} \\
\hline & AProVE & MU-TERM & $\mathrm{NaTT}$ & WANDA & NTI'08 & NTI'18 (select_lmne) \\
\hline $\mathcal{L}$ (173 TRSs) & 172 & 81 & 109 & 0 & 152 & 158 \\
\hline $\mathcal{U}(160$ TRSs $)$ & 0 & 0 & 0 & 0 & 4 & 6 \\
\hline
\end{tabular}

Table 4. Number of successes (NO) on $\mathcal{L}$ and $\mathcal{U}$ obtained during TC'17 and those obtained by NTI during our experiments.

\section{Conclusion}

We have reconsidered and modified the unfolding-based technique of [13] for detecting loops in standard term rewriting. The new approach uses disagreement pairs for guiding the unfoldings, which now are only partially computed, whereas the technique of [13] consists of a thorough computation followed by a mechanism for eliminating some rules. Two theoretical questions remain open: in terms of loop detection, is an approach more powerful than the other and does semiunification subsume matching and unification?

We have implemented the new approach in our tool NTI and compared it to [13] on a set of 333 rewrite systems. The new results are better (better times, more successful proofs, less unfolded rules). Moreover, the approach compares well to the tools that participated in TC'17. However, the number of generated rules is still important. In an attempt to reduce it, during our experiments we added the elimination mechanism of [13] to the new approach, but the results we recorded were not satisfactory (an equivalent, slightly smaller, number of generated rules but, due to the computational overhead, bigger times and less successes); hence, we removed it. Termination analysis may help to reduce the number of unfolded rules by detecting terminating SCCs in the estimated dependency graph i.e., SCCs on which it is useless to try a non-termination proof. In other words, we could use termination analysis as an elimination mechanism. Several efficient and powerful termination analysers have been implemented so far [16] and one of them could be called by NTI. A final idea to improve our approach would be to consider more sophisticated strategies for selecting disagreement pairs. 


\section{Acknowledgements}

The author thanks the anonymous reviewers for their many helpful comments and constructive criticisms. He also thanks Fred Mesnard for presenting the paper at the symposium.

\section{References}

1. M. Alpuente, M. Falaschi, G. Moreno, and G. Vidal. Safe folding/unfolding with conditional narrowing. In M. Hanus, J. Heering, and K. Meinke, editors, Proc. of Algebraic and Logic Programming, 6th International Joint Conference (ALP/HOA 97), volume 1298 of LNCS, pages 1-15. Springer, 1997.

2. AProVE Web site. http://aprove.informatik.rwth-aachen.de/

3. T. Arts and J. Giesl. Termination of term rewriting using dependency pairs. Theoretical Computer Science, 236:133-178, 2000.

4. F. Baader and T. Nipkow. Term Rewriting and All That. Cambridge University Press, 1998.

5. J. Giesl, C. Aschermann, M. Brockschmidt, F. Emmes, F. Frohn, C. Fuhs, J. Hensel, C. Otto, M. Plücker, P. Schneider-Kamp, T. Ströder, S. Swiderski, and R. Thiemann. Analyzing program termination and complexity automatically with AProVE. Journal of Automated Reasoning, 58(1):3-31, 2017.

6. J. Giesl, R. Thiemann, and P. Schneider-Kamp. Proving and disproving termination of higher-order functions. In B. Gramlich, editor, Proc. of the 5th International Workshop on Frontiers of Combining Systems (FroCoS'05), volume 3717 of LNAI, pages 216-231. Springer, 2005.

7. J. V. Guttag, D. Kapur, and D. R. Musser. On proving uniform termination and restricted termination of rewriting systems. SIAM Journal of Computing, 12(1):189-214, 1983.

8. D. Kapur, D. Musser, P. Narendran, and J. Stillman. Semi-unification. Theoretical Computer Science, 81(2):169-187, 1991.

9. C. Kop. WANDA - A higher-order termination tool. http://wandahot. sourceforge.net.

10. D.S. Lankford and D. R. Musser. A finite termination criterion. Unpublished Draft, USC Information Sciences Institute, Marina Del Rey, CA, 1978.

11. MU-TERM Web site. http://zenon.dsic.upv.es/muterm/

12. NaTT - The Nagoya Termination Tool. https://www.trs.css.i.nagoya-u.ac.jp/NaTT/.

13. E. Payet. Loop detection in term rewriting using the eliminating unfoldings. Theoretical Computer Science, 403(2-3):307-327, 2008.

14. StarExec - A cross-community solver execution and benchmark library service. http://www.starexec.org/

15. The Annual International Termination Competition. http://termination-portal.org/wiki/Termination_Competition.

16. Termination Portal - An (incomplete) overview of existing tools for termination analysis. http://termination-portal.org/wiki/Category:Tools

17. Termination Problems Data Base. http://termination-portal.org/wiki/TPDB

18. Y. Toyama. Counterexamples to the termination for the direct sum of term rewriting systems. Information Processing Letters, 25(3):141-143, 1987. 